\title{
Formación Inicial de Docentes desde una Perspectiva de Justicia Social: Una Aproximación Teórica
}

\author{
Initial Teacher Education from a Social Justice Perspective: A \\ Theoretical Approach
}

\section{Formação Inicial de Professores a partir de uma Perspectiva da Justiça Social: Uma Aproximação Teórica}

\author{
César Peña-Sandoval * \\ Carmen Montecinos
}

Pontificia Universidad Católica de Valparaíso

\begin{abstract}
A partir una caracterización del sistema escolar en Chile, que presenta altos índices de segregación social e inequidad, se argumenta la importancia de preparar profesores chilenos para enseñar desde una perspectiva de justicia social. Sleeter, Montecinos y Jiménez (2016) proponen un enfoque con cuatro temáticas a incluir en un currículo para la formación docente desde una perspectiva de justicia social: (a) situar a las familias y a las comunidades dentro de un análisis de las desigualdades estructurales; (b) desarrollar relaciones de reciprocidad con los estudiantes, las familias y las comunidades; (c) enseñar teniendo altas expectativas académicas en los estudiantes, capitalizando en su cultura, lengua e identidad; y (d) elaborar y enseñar un currículo que integre las perspectivas marginalizadas y enfrente de manera explícita los temas de equidad y poder. Estas dimensiones se elaboran teóricamente y luego se ejemplifica a través de las acciones, cogniciones y disposiciones de una estudiante de pedagogía quien en su practicum enseña desde una perspectiva de justicia social.
\end{abstract}

Descriptores: Educación para la justicia social, Formación inicial de docentes, Estudiantes vulnerables, Practicum.

\begin{abstract}
Beginning with a characterization of Chile's school system, which presents high levels of social segregation and inequity, the importance of preparing teachers from a social justice perspective is introduced. Sleeter, Montecinos y Jimenez (2016) have developed an approach which entails four issues to be addressed in a teacher preparation curriculum that has a social justice orientation: (a) situate families and communities within an analysis of structural inequalities; (b) develop relationships of reciprocity with students, families and communities; (c) teach with high expectations, capitalizing on students' culture, languages and identities; and (d) develop and teach a curriculum that integrates marginalized perspectives and directly addresses issues of equity and power. This approach is exemplified through the actions, cognitions and disposition of a student teacher who teaches from a social justice perspective.
\end{abstract}

Keywords: Social justice education, Initial teacher preparation, Vulnerable students, Student teaching.

*Contacto: cesar.pena@pucv.cl

ISSN: 2254-3139

www.rinace.net/riejs/

revistas.uam.es/riejs
Recibido: $\quad 16$ de septiembre 2016

$1^{\text {a }}$ Evaluación: 13 de octubre 2016

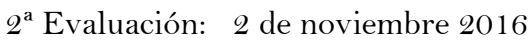

Aceptado: $\quad 15$ de noviembre 2016 


\begin{abstract}
A partir de uma caracterização do sistema escolar no Chile, que apresenta altos índices de segregação social e iniquidade, argumenta-se a importância de preparar professores chilenos para ensinar desde una perspectiva de justiça social. Sleeter, Montecinos e Jiménez (2016) propõem um enfoque com quatro temáticas a incluir em um currículo para a formação docente desde una perspectiva de justiça social: (a) situar as famílias e as comunidades dentro de uma análise das desigualdades estruturais; (b) desenvolver relações de reciprocidade com os estudantes, as famílias e as comunidades; (c) ensinar tendo altas expectativas acadêmicas nos estudantes, capitalizando sua cultura, língua e identidade; e (d) elaborar e ensinar um currículo que integre as perspectivas marginalizadas e enfrente de maneira explícita os temas de equidade e poder. Estas dimensões se elaboraram teoricamente e logo se exemplificam por meio de ações, cognições e disposições de um estudante de pedagogia que em sua prática ensina desde uma perspectiva de justiça social.
\end{abstract}

Palavras-chave: Educação para a justiça social, Formação inicial de professores, Estudantes vulneráveis, Prática.

\title{
Introducción
}

La evidencia en Chile nos señala que las instituciones en las cuales los docentes desempeñan sus funciones son espacios en los cuales los estudiantes de poblaciones marginalizadas por la pobreza experimentan exclusión social, con pocas oportunidades de movilidad social (Valenzuela, Bellei y De los Ríos, 2014). Remar contra un sistema educacional que reproduce inequidades sociales requiere docentes comprometidos con la justicia social y con competencias profesionales para que este compromiso se materialice en sus acciones cotidianas con los estudiantes, familias y colegas. El presente artículo tiene como objetivo analizar propuestas teóricas para la formación inicial de profesores desde una perspectiva de justicia social, focalizándose en un modelo desarrollado por Sleeter, Montecinos y Jiménez (2016).

A continuación, presentaremos una panorámica de las principales características del sistema educacional en Chile que revelan la importancia de considerar el contexto para el cual se prepara a un docente en el diseño de la formación de profesores orientada desde y hacia la justicia social (FDJS). Luego de examinar diversos referentes teóricos profundizamos en las cuatro dimensiones propuestas por Sleeter y otros (2016) para caracterizar la enseñanza para la justicia social. Las implicaciones prácticas de estas dimensiones se ilustran a través de datos producidos por el primer autor en un estudio más amplio sobre el desempeño de estudiantes de pedagogía en su practicum profesional (Peña-Sandoval, 2015). El artículo concluye con reflexiones respecto de cómo la formación inicial de profesores puede generar experiencias que propicien en los futuros maestros la capacidad para entender las desigualdades estructurales que generan dificultades para el éxito académico de estudiantes en situaciones de marginalidad, incorporando a las comunidades marginalizadas y los conocimientos que ellas han desarrollado en el diseño de un currículo y pedagogía culturalmente relevante que propicia altas expectativas de aprendizaje.

\section{Características del sistema escolar en Chile}

Durante los últimos 35 años las políticas públicas en Chile han propiciado un cuasimercado educacional en el cual la elección de los consumidores, la competencia entre centros escolares y un estado evaluador han buscado apalancar el mejoramiento de la calidad del sistema (Maroy, 2004). Como efectos de estas políticas, el sistema escolar en 
Chile presenta altos niveles de cobertura en un contexto de altos niveles de segregación social y de inequidad (OECD, 2015; Valenzuela et al., 2014). Hay cuatro características del sistema escolar que develan los retos clave que requieren abordar las instituciones formadoras de docentes para que, en este contexto, sus egresados ofrezcan una educación con calidad y equidad.

Primero, el estado ha asumido un rol de subsidio a la demanda buscando garantizar que las familias puedan elegir entre cuatro tipos de establecimientos educacionales: (a) centros escolares municipales, públicos, administrados por los municipios y financiados por un subsidio basado en la asistencia de los estudiantes; (b) centros escolares privados que también reciben el mismo subsidio; (c) centros escolares municipales cuya administración ha sido delegada a una corporación privada y también se financian a través del subsidio estatal; y (d) centros escolares privados financiados enteramente por las familias. La participación del sector privado en el mercado educacional de Chile ha crecido mientras la participación del sector público ha disminuido. Una consecuencia de esto es que Chile presenta altos niveles de cobertura tal que el $94 \%$ de los estudiantes menores de 13 años asiste a un centro escolar, cifra que alcanza sobre el $70 \%$ en la educación secundaria. Otra consecuencia es una merma sostenida de la matrícula en el sector público: 39\% para el año 2011, cifra que al año 2016 llega a $36 \%$.

Segundo, los estudiantes chilenos asisten a centros escolares con pares de su mismo grupo socio-económico, lo que se expresa en una alta segregación social (OECD, 2013). En los establecimientos públicos, el $80 \%$ de los estudiantes provienen de familias del nivel socio económico bajo o medio bajo, mientras que el 20\% de los estudiantes en establecimientos privados subvencionados provienen de estos grupos (García Huidobro, 2010). La segregación social es mayor en la enseñanza secundaria en comparación a la primaria (Valenzuela et al., 2014). En los resultados PISA 2012 Chile se encuentra entre los países con mayor correlación entre el origen social del estudiante y el nivel de logro en la prueba de matemática (OECD, 2015). La segregación se asocia al modelo de mercado que posibilita a las familias a elegir según su capacidad de pago y a los colegios particulares a seleccionar a los estudiantes según sus antecedentes académicos y las posibilidades de co-pago de las familias.

Tercero, existe un currículo nacional que, con diversos grados de ajustes a condiciones locales, debe ser implementado en todos los establecimientos educacionales del país. Los estudiantes rinden pruebas censales en cuarto y octavo año de la primaria y en segundo año de la secundaria que miden la calidad de la educación que imparten los establecimientos (SIMCE). Esta prueba tiene altas consecuencias para el centro escolar por cuanto determina la percepción de prestigio en la sociedad y porque determina el nivel de autonomía con el cual ejerce respecto de la supervisión del Ministerio de Educación.

En base a los resultados en el SIMCE existe la percepción ciudadana de que los centros escolares públicos son de inferior calidad en comparación a los privados. No obstante, después de controlar por diferencias socioeconómicas de los estudiantes, la evidencia muestra que los resultados del SIMCE no difieren estadísticamente entre centros escolares públicos y privados (Manzi, Strasser, San Martín y Contreras, 2008). La baja reputación de los centros escolares públicos lleva a que estos sean el destino de quienes, por diversas limitaciones financieras o académicas/conductuales, no son atractivos para 
los centros escolares privados (los centros escolares públicos no pueden seleccionar) o no pueden trasladarse a otros lugares de la ciudad (Elacqua, 2012).

Cuarto, la arquitectura del sistema escolar se estructura en ocho años de enseñanza básica y cuatro años de enseñanza secundaria, para un total de 12 años de escolarización obligatoria. Así, salvo algunas excepciones, como por ejemplo educación física, los profesores se forman para ejercer en el nivel preescolar o en el nivel primario o en el nivel secundario. Los profesores primarios se forman como generalistas y los de secundaria como especialistas disciplinarios.

En síntesis, los docentes chilenos que optan por trabajar en los centros escolares públicos trabajarán mayoritariamente con estudiantes creciendo en las comunidades con menor nivel socio económico, trabajarán en centros escolares con bajo prestigio social y deberán enseñar un currículo nacional exigente y bajo un fuerte sistema de rendición de cuentas a través del SIMCE. Las instituciones que forman a profesores necesitan tomar estas variables del contexto laboral en consideración al momento de diseñar su currículo. En este contexto de desigualdad y segregación que padecen muchos sistemas educativos, no solo el chileno, en las últimas décadas hemos visto una creciente atención a conceptos como la enseñanza para la diversidad y la justicia social.

\section{Marco conceptual}

Tal como diversidad se convirtió en un término de moda (Ladson-Billings, 1999), justicia social ha ganado terreno en el panorama educativo, pero aún no alcanza un consenso en torno a su significado (Carlisle, Jackson y George, 2006; Murillo y Hernández-Castilla, 2011; North, 2008). Dentro de los notables esfuerzos que avanzan en su teorización y precisión encontramos a Ayers, Quinn y Stovall (2009), quienes sostienen que "la educación para la justicia social se basa en tres principios: (1) la equidad, el principio de justicia...; (2) el activismo, el principio de la agencia...; [y] (3) la alfabetización social, el principio de relevancia” (p. xiv). Por su parte, Carlisle et al. (2006) la definen "como la combinación consciente y reflexiva de contenidos y procesos destinados a mejorar la equidad en grupos con múltiples identidades sociales (por ej., raza, clase, género, orientación sexual, capacidades), favorecer las perspectivas críticas y promover la acción social” (p. 57).

En el marco de la formación inicial de profesores, Cochran-Smith y otros (2009) destacan la noción de "buena y justa enseñanza" (good and just teaching) sustentada en los hallazgos de programas de formación con agendas explícitas de justicia social, donde los futuros profesores "entendieron este concepto y lo aplicaron en las salas de clase y en las oportunidades de aprendizaje que crearon para sus estudiantes" (p. 348). Además, para contrarrestar la ambigüedad y el aparente vacío de significado de la Formación Docente para la Justicia Social (FDJS), Cochran-Smith y otros (2009) subrayan que, en un contexto donde muchos programas que declaran un enfoque de justicia social, la FDJS no debe ser entendida como mera retórica ideológica; por el contrario, es una perspectiva que pone su foco en el aprendizaje escolar, teniendo la mejora de las oportunidades de aprendizaje y de vida de los estudiantes como su preocupación central.

Así, elementos clave en la preparación de futuros profesores orientados por la justicia social son el dominio disciplinario, el conocimiento pedagógico del contenido y los métodos y estrategias didácticas para trabajar con sus estudiantes. Además, son los 
marcos teóricos de interpretación, los aspectos sociales, intelectuales y organizacionales que preparan a los futuros profesores para desempeñarse en escenarios escolares diversos. Por último, Cochran-Smith y otros (2009) agregan la capacidad para trabajar con las familias, los pares y las comunidades de base. Por lo tanto, la FDJS implica una preparación que no solo promueve equidad y calidad en los aprendizajes, sino también prácticas más democráticas y colaborativas con las comunidades.

En el concierto hispano y latinoamericano también se ha relevado la necesidad de una educación más justa, particularmente en un contexto de crecientes desigualdades socioeconómicas como los que caracterizan a la región (p. ej., Belavi y Murillo, 2016; Murillo y Hernández-Castilla, 2011; Peña-Sandoval, 2011; Tedesco, 2012, Williamson y Montecinos, 2011). Junto con subrayar los conceptos de equidad educativa, igualdad de oportunidades, derechos humanos y atención a la diversidad, subrayan que la escuela que aspira a contribuir a la justicia social busca una educación donde todos y todas aprendan, logren un desarrollo integral y donde la democracia y la valoración de la multiculturalidad sean metas esenciales.

Atendiendo a los desarrollos arriba mencionados y situándonos en el contexto chileno, entendemos que el propósito de FDJS es promover transformaciones que aborden las necesidades de una población estudiantil cada vez más diversa, con especial foco en niños y jóvenes donde el factor central de su marginalización es la pobreza (Gorski, 2013). La población descendiente de pueblos originarios en Chile bordea el 10\% y el creciente influjo de poblaciones inmigrantes a Chile también pone nuestra atención en estudiantes minorizados (Shields, Bishop y Mazawi, 2005). En este manuscrito elaboramos el trabajo desarrollado por Sleeter et al. (2016), quienes luego de examinar diversos marcos teóricos referidos a la FDJS y sus principios, sintetizaron su propuesta en las siguientes cuatro dimensiones a considerar en el proceso formativo de los docentes:

- Situar a las familias y a las comunidades dentro de un análisis de las desigualdades estructurales;

- Desarrollar relaciones de reciprocidad con los estudiantes, las familias y las comunidades;

- Enseñar teniendo altas expectativas académicas en los estudiantes, capitalizando en su cultura, lengua, experiencia e identidad;

- Elaborar y enseñar un currículo que integre las perspectivas marginadas y enfrente de manera explícita los temas de equidad y poder.

Primero, situar las familias y las comunidades dentro de un análisis de las desigualdades estructurales implica que los profesores, al trabajar con estudiantes de contextos vulnerables, no adopten una postura centrada en el déficit (Chubbuck, 2010). La perspectiva de déficit estereotipa a las familias pobres (Gorski, 2013), incluso haciendo que estas internalicen dichos estereotipos (Jones y Vagel, 2013). Por el contrario, se propone que los profesores sitúen a los estudiantes y las familias en el marco de múltiples relaciones desiguales en términos sociales, económicos y de poder. Esto porque son las relaciones desiguales de poder las que limitan el acceso a los recursos de la sociedad generando exclusión y marginalidad. Por tanto, adoptar la perspectiva de la justicia social implica que, en lugar de ver la pobreza como fracaso personal, esta sea comprendida como efecto de políticas y sistemas injustos (Jones y Vagel, 2013). Desde 
esta perspectiva, los profesores -en formación y en ejercicio- pueden identificar las barreras que existen al interior de los centros escolares y el aula, así como la resiliencia y las potencialidades intelectuales de los estudiantes. Esta dimensión implica convertirse en defensores de los estudiantes dentro y fuera de los centros escolares, y ser aliados en lugar de antagonistas de las familias de las que provienen (Sleeter et al., 2016).

En segundo lugar $-\mathrm{y}$ siendo un aspecto menos enfatizado la literatura- se destaca la capacidad de los profesores para desarrollar relaciones de reciprocidad con los estudiantes y las familias, especialmente aquellas que provienen de sectores marginados. Se propone superar la preconcepción de que es normal que existan malas relaciones entre educadores y comunidades pobres que frecuenten; es decir, aprender a "trabajar con (no contra) los individuos, las familias y las comunidades" (Cochran-Smith, 2004, p. 72). Por su parte, Gorski (2013) explica que el desarrollo de este tipo de relaciones requiere esfuerzo y sugiere comenzar por la construcción de relaciones de confianza y reciprocidad con los estudiantes. En la medida en que los profesores acojan las preocupaciones de los estudiantes y las tomen en serio, ganarán su confianza. Por tanto, es importante trabajar constantemente para llegar a la comunidad, tratándola como socios necesarios en la educación de los niños y jóvenes.

Esta idea de mayor horizontalidad en las relaciones de los centros escolares con las comunidades a las que sirven también se encuentra, por ejemplo, en los trabajos de Zeichner (2009, 2010) y Zeichner y Payne (2013). Allí, se apuntan a una formación inicial que prepara docentes competentes para conectarse de manera auténtica con la cultura, necesidades e intereses de las familias y las comunidades de los niños y jóvenes que han de servir. Por tanto, el conocimiento de las comunidades se vuelve parte del proceso de formación docente. Este enfoque resalta la necesidad de una distribución del conocimiento más horizontal, donde los profesores en ejercicio y los miembros de la comunidad son considerados como co-formadores docentes pues su saber es tan importante como el que proviene de la universidad. Este tipo de trabajo involucra la creación de espacios híbridos (Zeichner, 2010) donde la universidad, los centros escolares y la comunidad, en una relación democrática, trabajan mancomunadamente con el propósito compartido de dar mejores oportunidades de aprendizaje a los futuros profesores. Finalmente, a través de estas relaciones horizontales, los programas de formación inicial avanzan desde el discurso hacia la práctica de la perspectiva de justicia social.

Tercero, enseñar teniendo altas expectativas académicas en los estudiantes, aprovechando su cultura, lengua, experiencia e identidad, implica que los profesores construyan sobre los recursos intelectuales que traen los estudiantes. Llevando a cabo un andamiaje cuidadoso saben cómo superar brechas entre sus conocimientos y habilidades y las metas de aprendizaje que propone el currículo escolar. Por ejemplo, el enfoque de la Pedagogía Culturalmente Relevante (PCR) (Gay, 2010; Ladson-Billings, 1995) invita a los profesores a iniciar el proceso de enseñanza en el nivel en que están los estudiantes, atendiendo cuidadosamente a las concepciones y comprensiones previas de los estudiantes, apoyándolos y animándolos para un aprendizaje cada vez más complejo. Además, autores como Cochran-Smith (2004) y Gorski (2013) reafirman esta postura que subraya la tarea de potenciar el desarrollo de un pensamiento de orden superior sin asumir que el rendimiento académico actual de los estudiantes desfavorecidos indica su potencial de aprendizaje. Por el contrario, los profesores orientados por la justicia social reconocen que la cultura y la lengua de los estudiantes son fundamentales en términos 
del aprendizaje y comprenden que valorar estos aspectos es valorar la identidad del estudiante.

Finalmente, crear y enseñar un currículo que integre las perspectivas marginadas y enfrente de manera explícita los temas de equidad y poder encierra la posibilidad de desarrollar activismo democrático. La educación para la justicia social es desarrollar una enseñanza que prepara a los jóvenes para analizar y cuestionar las formas de discriminación existentes, que ayuda a comprender la naturaleza y las manifestaciones de la opresión social y que proporciona estrategias de intervención para el desarrollo del pensamiento y la acción liberadora (Carlisle et al., 2006). Cochran-Smith (2004) explica que "parte de aprender a enseñar para la justicia social es luchar para hacer visibles y explícitas -al nivel que sea apropiado para el desarrollo de los estudiantes, las desigualdades de la sociedad y las estructuras institucionales en las que están inmersos" (p. 78). En términos de la PCR, equivale al principio de consciencia sociopolítica (LadsonBillings, 2006) a través del cual los profesores conectan las desigualdades sociales y los temas de clase, género, etnia y raza, entre otros, incorporándolos en su enseñanza cotidiana. Consecuentemente, los profesores ayudan a los estudiantes a utilizar las distintas habilidades que aprenden para comprender mejor su posición social y criticar su contexto.

\section{Implicancias para la práctica docente de las dimensiones de la enseñanza para la justicia social}

A continuación, ejemplificamos cómo se pueden desplegar en la docencia del aula secundaria cada una de estas dimensiones. Con este propósito hemos seleccionado datos de un caso, Paula Cádiz, que ilustran de mejor manera cómo las dimensiones del modelo propuesto por Sleeter y otros (2016) se pueden materializar en las interacciones pedagógicas y en las cogniciones y valores de docentes que enseñan desde una perspectiva de justicia social. Paula, de 23 años, estudiaba para ser profesora de Historia en la escuela secundaria y participó en un estudio de caso múltiple más amplio (PeñaSandoval, 2015) cuyo objetivo general fue conocer los aprendizajes contextuales (institución y trasfondos socioculturales de los estudiantes) construidos por los profesores en formación en su practicum y cómo estos eran incorporados en el desempeño en el aula. El estudio utilizó un muestreo intencionado (Merriam, 2009), seleccionando siete casos a partir del juicio de los formadores universitarios respecto de profesores en formación destacados por su orientación reflexiva y de justicia social.

De entre los siete casos del estudio citado, Paula destacó por su perspectiva y desempeño al enseñar en un curso del primer año de secundaria en un colegio municipal con una alta proporción de estudiantes vulnerables. Ella se educó en un liceo municipal, público, y provenía de un barrio de clase media, media baja. Previo a estudiar la carrera de Pedagogía, mientras completaba su licenciatura en Historia, trabajó como voluntaria en un programa de preparación preuniversitaria para jóvenes de barrios marginalizados. Ahí desarrolló experiencias y conocimientos como educadora popular.

Luego de firmar un consentimiento informado, las técnicas de producción de datos incluyeron: a) entrevista inicial semi-estructurada, grabada, previo al inicio del practicum; b) tres observaciones de clases de 90 minutos cada una espaciadas en un periodo de cuatro meses; y c) una segunda entrevista, grabada, al final del período de 
observación. Las entrevistas duraron entre 60 y 90 minutos, fueron transcritas y junto con el registro ampliado de cada observación se analizaron a partir de la teoría fundamentada (Bryant y Charmaz, 2007) utilizando la técnica de análisis del contenido (Denzin y Lincoln, 2005).

El programa aludido se imparte en una universidad tradicional, pública y laica de Santiago de Chile a licenciados de distintas disciplinas , quienes obtendrán el título de Profesor en Enseñanza Media (nivel secundario). Dicho programa se caracteriza por fomentar una educación pública, justa y de calidad para todos y todas las estudiantes. El programa prioriza que el practicum se lleve a cabo en escuelas municipales o privadas subvencionadas que sirven a estudiantes en situación de pobreza. Se espera que los profesores en formación puedan desarrollar una perspectiva reflexiva y crítica, atenta a los contextos y orientada a la acción pedagógica transformadora. El estudio no tuvo como objetivo evaluar la calidad del programa.

\subsection{Conciencia de las desigualdades estructurales}

Situar a las familias y comunidades de los estudiantes dentro de desigualdades sistémicas supone que los profesores superan la visión del déficit y de los estereotipos cuando enseñan a estudiantes en contextos de pobreza. En el caso de Paula, se evidencia una clara conciencia al respecto, reconociendo que los estudiantes y sus contextos empobrecidos responden a características estructurales del sistema educativo y de la sociedad. Su biografía y sus experiencias previas en educación popular en barrios marginalizados aparecen como los cimientos vivenciales de una posición crítica y reflexiva ante la realidad social. Por ejemplo, ante fenómenos como la deserción escolar o la falta de oportunidades, ella ha construido una perspectiva de justicia social que se expresa en el desarrollo de una conciencia sociopolítica (Ladson-Billings, 2006):

En contextos vulnerables, yo siento que la idea es disminuir lo que más se pueda lo que es el ausentismo... Allá [en otro centro de escolar en el cual realizó un practicum] tratando el tema de la crisis económica, le pregunté a [un niño] “qué harías en el lugar de la familia que estaba en medio de la crisis económica?”. Y él me respondió algo como... "bueno, yo trabajaría, pero en verdad yo nunca voy a salir de la pobreza" ... Entonces, como sujeto activo e histórico, siento que es muy difícil, porque es como si las cosas están dadas... yo siento que no hay que detenerse, que no hay que bajar los brazos tampoco, hay que seguir dándole. (Entrevista pre-observación)

Tal como lo establecen diversos autores (p. ej., Chubbuck, 2010; Gorski, 2013), muchos profesores que enfrentan este tipo de contextos y dificultades en sus aulas suelen elaborar explicaciones que refieren a la existencia de una supuesta "cultura de la pobreza", culpando a las familias o a los propios estudiantes por ser los causantes de su fracaso escolar (North, 2008). Por el contrario, quienes están orientados por principios de justicia social rechazan dichos estereotipos y -como Paula- descartan la supuesta incapacidad de los estudiantes, reconociendo que las barreras están ya planteadas por contexto sin oportunidades. Siguiendo a Howard (2006) Paula encarna una pedagogía trasformacionista, que desafía la dinámica de segregación social y del sistema escolar y está dispuestos a asumir el desafío profesional que ello le plantea.

\subsection{Relaciones de reciprocidad}

Una de las preguntas de estudio en el cual participó Paula interrogó por cómo se manifestaba la orientación de justicia social en la manera en que los profesores en 
formación piensan, planifican y ejecutan la enseñanza en su practicum. Los datos recopilados en torno a Paula refrendan la segunda dimensión de Sleeter y otros (2016) respecto del tipo de relaciones que ella construyó con sus estudiantes. Reconociendo el carácter fundamental del conocimiento del contenido disciplinario, del conocimiento pedagógico y de la gestión del aula, ella destacó que la construcción de relaciones auténticas es la base que posibilita un buen desempeño docente y el aprendizaje de los estudiantes. La reciprocidad también implica validación por parte de los estudiantes, para quienes Paula era una docente cercana, a quien reconocen como parte de su mundo:

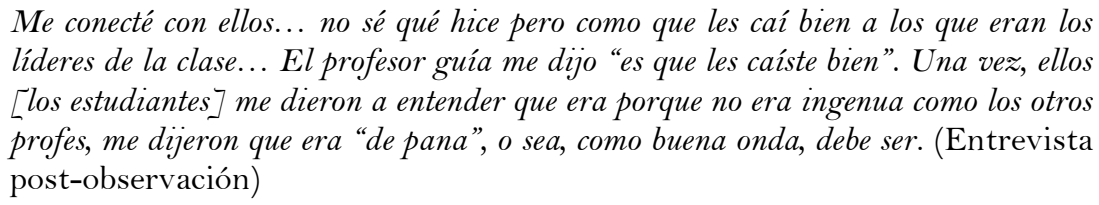

Al validar la cultura y el lenguaje de los estudiantes, Paula estableció un vínculo auténtico, ejemplificando el principio del cuidado presente en la pedagogía culturalmente relevante (Gay, 2010). Los profesores no solo ponen atención al rendimiento académico, sino también a la condición humana de los estudiantes. Este cuidado abarca sentimientos de preocupación o cariño, a la vez que tiene dimensiones emocionales, intelectuales, éticas, de confianza, de acción y de responsabilidad por los logros académicos. Al mismo tiempo, el cuidado por los estudiantes (su cultura, necesidades e intereses) es también un reconocimiento de sus trasfondos familiares y comunitarios, especialmente cuando se trata de estudiantes en situación de pobreza.

\subsection{Expectativas académicas y uso pedagógico de conocimiento del contexto}

La tercera dimensión de Sleeter y colaboradores (2016) señala que los profesores orientados a la justicia social implementan una enseñanza que apela al mayor potencial intelectual de los estudiantes, lo cual está marcado por el acceso y uso de los aspectos contextuales (cultura, lengua, experiencias, identidad, etc.). Paula llegó con altas expectativas académicas, las cuales se expresaron en la intención de implementar clases con contenidos disciplinarios ambiciosos y actualizados, propios de su reciente formación universitaria. Al evaluar los conocimientos previos de los estudiantes o su punto de partida, tomó decisiones que le permitieron adaptar sus contenidos y métodos. Paula no desechó la idea de enseñar un currículo ambicioso, pero tomó conciencia de la necesidad de disponer de andamiajes para que el contenido curricular fuera accesible a sus estudiantes. Las expectativas no bajaron, pero la trayectoria para lograrlas implicaba enseñar cosas que no tenía previamente contempladas como docente de historia:

$$
\begin{aligned}
& \text { Les enseño que tienen que escribir, porque hay muchas palabras difíciles en historia y } \\
& \text { que yo no sabía que eran tan desconocidas para ellos... Más aún, cuando empecé a } \\
& \text { ver La Revolución Rusa, ya revolución era una palabra complicada porque la } \\
& \text { escriben mal. Por ejemplo, la palabra proletariado, yo no sabía que era tan difícil, } \\
& \text { pero para los chicos es muy difícil... Se demoran mucho en escribir tres palabras, } \\
& \text { pero yo quiero que escriban. (Entrevista post-observación) }
\end{aligned}
$$

Tener altas expectativas académicas para los estudiantes es una actitud docente esencial dentro del enfoque de justicia social. Sin embargo, tales expectativas están mediadas por el contexto sociocultural y el tipo de centro escolar. En consecuencia, esta dimensión se verifica de manera situada en las prácticas de quien internaliza auténticamente los contextos. Tal como lo han señalado diversos autores (p. ej., Cochran-Smith, 2004; Gorski, 2013), quienes manifiestan una postura de justicia en la enseñanza aspiran al 
desarrollo de un pensamiento profundo en sus estudiantes, proponiendo metas de aprendizaje que parten desde el nivel inicial de quienes aprenden. Si bien se ha señalado que quienes abrazan este enfoque y trabajan con estudiantes que viven en pobreza no deben asumir que el rendimiento actual de los estudiantes indica su potencial de aprendizaje, también es necesario añadir -tal cual lo expresa Paula- que el punto de partida y la trayectoria de aprendizaje para alcanzar la meta varían según cada contexto.

\subsection{Explicitar las desigualdades e integrar las perspectivas marginalizadas al curricular}

De acuerdo a la literatura, entre las creencias que manifiestan los profesores orientados a la justicia social encontramos, por ejemplo: que los asuntos relacionados con las desigualdades deben ser discutidos abiertamente en la sala de clases; que es responsabilidad del profesor desafiar las prácticas y normativas escolares que mantienen las inequidades sociales; y que se debe enseñar a los estudiantes a pensar críticamente acerca de su realidad y las políticas educativas que les afectan (Cochran-Smith, Ludlow, Ell, O'Leary y Enterline, 2012). Paula ejemplifica dichas creencias tanto en entrevistas como en episodios de clase registrados. Aunque la perspectiva de justicia social no se manifestó en la creación de contenido curricular, sí tuvo lugar en la incorporación de las perspectivas marginadas en discusiones y actividades de clase para las que mostró autonomía de creación. En el marco del currículo oficial de Historia, Paula innovó integrando temas como la pobreza y las relaciones de poder. Al ser consultada por el resultado de su propuesta de clase en el marco de una de las sesiones registradas, señaló:

$$
\begin{aligned}
& \text {...estábamos viendo el concepto de crisis y yo pedí que lo relacionaran con algo que } \\
& \text { les haya pasado, que ellos hayan sentido como que era una crisis... Christopher me } \\
& \text { escribió “mi crisis fue cuando fui a robar y me llevaron en cana [a prisión] y estuve } \\
& \text { en el calabozo”... Dante va a ser padre en un mes más y él es chico, está en primero } \\
& \text { medio y le va muy mal... y yo ¿̇qué le voy a decir?, ¿̇que siga estudiando, que se } \\
& \text { quede en el colegio? No pues, él necesita plata [dinero] para mantener a la guagua } \\
& \text { [el bebé]... y que se ponga a trabajar nomás y que desde ahora ya esté en un trabajo } \\
& \text { part-time. (Entrevista post-observación) }
\end{aligned}
$$

Nuevamente, Paula explicita su conciencia sociopolítica (Ladson-Billings, 2006) al planificar la enseñanza y sopesar el contexto en el cual la ejerce. Esto se potencia con la incorporación de elementos contextuales que se vuelven contenido curricular "no oficial" toda vez que permite a los estudiantes expresar opiniones sobre temas que les afectan directamente (p. ej., marginalidad). Al explicitar temas como la pobreza y aquellos propios de la contingencia social, Paula fortalece la construcción del vínculo y trae el "mundo de la vida" al currículo escolar. Esta integración de perspectivas marginadas expresa una postura ética que asume riesgos ante temas controversiales, reflejando un activismo sobre los mismos y buscando dar oportunidades a sus estudiantes para desarrollar un pensamiento y acción liberadores para analizar y cuestionar diversas formas de discriminación u opresión (Carlisle et al., 2006).

Finalmente, la integración de perspectivas marginadas también implica el uso de estrategias y recursos pertinentes para conectar con los trasfondos socioculturales de los estudiantes, con el potencial de alcanzar su entorno más amplio (familias, comunidades). Un recurso muy utilizado por Paula en sus clases es el audiovisual, particularmente el vídeo y las imágenes. Al respecto Gorski (2013) ha subrayado la relevancia de trabajar con recursos que favorecen la creación artística en contextos de pobreza pues se ha demostrado su potencial de explicitación de conocimientos previos y facilitadores de 
aprendizaje. El siguiente extracto se refiere a la reflexión de Paula en torno a la estrategia y recursos utilizados por ella en el liceo municipal donde enseña:

\begin{abstract}
Surgió la idea de pedirles un trabajo grupal en donde ellos tenían que imaginar que viajaban por América Latina. Al final tenían que hacer un vídeo contándome cómo fue su viaje... La primera parte era entregar un escrito de cómo había sido su viaje, combinando datos reales con la imaginación. Algunos se sacaron fotos con maletas, otros hicieron Photoshop. Ellos hicieron los videos, después los vimos todos en grupo y luego lo evaluamos en conjunto. (Entrevista post-observación)
\end{abstract}

Al proponer el uso de las nuevas tecnologías para un viaje imaginario, Paula evidenció los problemas de accesibilidad a viajes reales (explicitar las desigualdades), junto con incentivar la incorporación de elementos propios de la cultura de los estudiantes para narrar su viaje ideal (integrar perspectivas marginadas). Paula ofreció a los estudiantes un espacio para demostrar competencia en el uso de las tecnologías y, al mismo tiempo, reflexionar sobre las condiciones materiales que limitan o posibilitan otros mundos. Ejemplificando estas dimensiones, Paula se mostró atenta a los elementos que facilitan el aprendizaje, ya sea desde los intereses de los que aprenden como desde el ambiente que condiciona las maneras de acercarse a la construcción de conocimientos.

\title{
4. Conclusiones
}

Siguiendo la discusión de Murillo y Hernández-Castilla (2011) sobre conceptualizaciones de justicia social, las dimensiones propuestas por Sleeter y otros (2016) y ejemplificadas en las acciones y cogniciones de Paula Cádiz dan cuenta de la importancia de preparar a los futuros docentes para: (a) conocer y utilizar los recursos culturales y capacidades que están presentes en las comunidades a las que sirve el centro escolar; (b) reconocer y valorar las diferencias culturales e individuales como una fortaleza y recurso pedagógico en el aula; (c) promover relaciones justas entre personas y colectivos sociales; (d) promover la participación de las familias y los estudiantes en las decisiones que afectan su escolarización; y (e) ampliar las capacidades de los estudiantes para ejercer su libertad de elección y contribución como un actor en la construcción de una sociedad más democrática.

En un contexto de inequidad y segregación social, los estudiantes que crecen situación de vulnerabilidad social y exclusión necesitan profesores como Paula que validan sus mundos, expandiendo su liberad al abrirles las puertas para conocer e imaginar otros mundos posibles. No utilizamos el ejemplo de Paula para argumentar que al utilizar la pedagogía para la justicia social se pueden compensar las grandes inequidades estructurales de Chile. Más bien nuestro argumento es que enseñar desde una perspectiva de justicia social puede marcar una diferencia en cómo los jóvenes marginalizados construyen su participación en la escuela, su visión de sí mismos en ese espacio social y su visión de futuro (Riley, 2013; Sleeter, 2011). El ejemplo de Paula tampoco es analizado para determinar la efectividad del programa de formación docente en el cual participó o cómo interactúa su biografía con las experiencias formativas diseñadas por este programa. Sobre ambos aspectos especulamos en estas conclusiones, pero abordarlos a cabalidad requiere de investigaciones diseñadas con esos propósitos.

La justicia, nos señala Barad (2007), es necesariamente sobre nuestras relaciones y responsabilidades mutuas. La orientación hacia la justicia social en los docentes se manifiesta discursiva y prácticamente a través de la construcción de relaciones con sus 
estudiantes y la incorporación del contexto como un recurso para la enseñanza. Paula enfatiza la necesidad de que la relación pedagógica sea construida a partir del vínculo personal (emocional o afectivo). En otras palabras, la relación humana hace posible y precede al proceso de enseñanza-aprendizaje. Con esta convicción, los profesores que enseñan desde una perspectiva de justicia social se orientan a (re)conocer a sus estudiantes, valoran los recursos culturales que ofrece el entorno comunitario de la escuela y luego incorporan estos conocimientos en la planificación e implementación de la enseñanza.

En Paula, sin embargo, no se evidencia la convicción de que ella, además de tener un vínculo con sus estudiantes, necesita vincularse con las familias y comunidades de las cuales ellos provienen. Para desarrollar relaciones de reciprocidad, se hace necesario el uso de recursos y estrategias que acercan el mundo (p. ej., familias, barrios, etc.) de los estudiantes al currículo escolar. A partir de la incorporación a las actividades de aprendizaje del contexto que van conociendo, los profesores van validando el conocimiento y los intereses generados por las comunidades marginalizadas. Es necesario destacar que este programa de formación inicial para docentes de secundaria (como muchos otros en Chile) no contempla trabajo en terreno con familias y centros comunitarios. En el caso de la universidad en la cual se formó Paula, la necesidad de conocer el contexto de los estudiantes se satisface indirectamente a través metodologías narrativas o dinámicas grupales que se comparten y potencian en los talleres de indagación sobre la práctica o en las didácticas de especialidad.

Otros autores han justificado la importancia de actividades formativas donde los futuros profesores tienen contacto directo con comunidades marginalizadas para aprender de ellas (Aramburuzabala, 2013; González, Moll y Amanti, 2005). Realizar visitas a las familias y realizar actividades de aprendizaje en servicio en los centros comunitarios, por ejemplo, aportaría un aprendizaje experiencial para una formación más sólida en términos de los saberes y otros recursos disponibles en el entorno a ser incorporados en el proceso pedagógico. La recomendación es que los programas integren estos espacios formativos para que los futuros profesores estén preparados para los desafíos que presentan los contextos más complejos. Las experiencias previas de Paula como educadora popular que marcan fuertemente su orientación hacia la justicia social y su capacidad de conectarse con los jóvenes refuerzan la importancia de ofrecer la formación inicial de profesores en las aulas universitarias, así como fuera de ellas.

El presente manuscrito es un ensayo con una selección de datos empíricos que permiten visualizar el correlato pedagógico de las dimensiones teóricas propuestas por Sleeter et al. (2016). El desarrollo de investigaciones que indaguen en los factores que posibilitan la adopción de una perspectiva de justicia social tanto en los discursos como en las acciones de los futuros docentes aportará a enriquecer esta propuesta. En efecto, la investigación puede centrarse, por ejemplo, en las diferencias en concepciones, creencias y significados que los futuros profesores construyen en torno a la enseñanza para la justicia social, así como también en cómo los programas de formación que declaran una perspectiva de justicia social la encarnan a través de sus actividades curriculares (formación teórica y formación práctica/clínica). Por otra parte, el caso de Paula levanta interrogantes respecto de las experiencias de socialización que los futuros docentes traen a la formación inicial y cómo estas interactúan con las experiencias de socialización profesional diseñadas por el currículo de formación. Estudios longitudinales que muestren la evolución de los futuros docentes en cuanto a sus acciones, cogniciones y 
disposiciones a medida que progresan en su preparación pueden entregar pistas respecto de la efectividad de las actividades de aprendizaje en el logro de las dimensiones propuestas por Sleeter et al. (2016) o por otros modelos de formación docente para la justicia social.

\section{Agradecimientos}

Los autores agradecen el apoyo financiero de Fondecyt Proyecto Postdoctorado (Nro. 3160530) y de CONICYT FB 003 en la preparación de este manuscrito.

\section{Referencias}

Aramburuzabala, P. (2013). Aprendizaje-servicio: Una herramienta para educar desde y para la justicia social. Revista Internacional de Educación para la Justicia Social (RIEJS), 2(2), 5-11.

Ayers, W., Quinn, T. y Stovall, D. (Eds.). (2009). Handbook of social justice in education. Nueva York: Routledge.

Barad, K. M. (2007). Meeting the universe halfway: quantum physics and the entanglement of matter and meaning. Durham, NC: Duke University Press.

Belavi, G. y Murillo, F. J. (2016). Educación, democracia y justicia social. Revista Internacional de Educación para la Justicia Social (RIEJS), 5(1), 13-34.

Bryant, A. y Charmaz, K. (2007). The SAGE handbook of grounded theory. Los Ángeles, CA: SAGE.

Carlisle, L. R., Jackson, B. W. y George, A. (2006). Principles of social justice education: the social justice education in schools project. Equity $\mathcal{E}^{2}$ Excellence in Education, 39, 55-64. doi:10.1080/10665680500478809

Chubbuck, S. (2010). Individual and structural orientations in socially just teaching: Conceptualization, implementation, and collaborative effort. Journal of Teacher Education, 61(3), 197-2 10. doi:10.1177/002248710935977

Cochran-Smith, M. (2004). Walking the road: Race, diversity, and social justice in teacher education. Nueva York: Teachers College Press.

Cochran-Smith, M., Shakman, K., Jong, C., Terrell, D. G., Barnatt, J. y McQuillan, P. (2009). Good and just teaching: the case for social justice in teacher education. American Journal of Education, 115, 347-377.

Cochran-Smith, M., Ludlow, L., Ell, F., O'Leary, M. y Enterline, S. (2012). Learning to teach for social justice as a cross-cultural concept: findings from three countries. European Journal of Educational Research, 1(2), 171-198. doi:10.12973/eu-jer.1.2.171

Denzin, N. K. y Lincoln, Y. S. (2005). The SAGE handbook of qualitative research. Thousand Oaks, CA: Sage Publications.

Elacqua, G. (2012). The impact of school choice and public policy on segregation: evidence from Chile. International Journal of Educational Development, 32, 444-453. doi:10.1016/j.ijedudev.2011.08.003

García-Huidobro, J. E. (2010). Para hacer pública la educación pública. En S. Martinic y G. Elacqua (Eds.), ¿̈Fin de ciclo? Cambios en la gobernanza del sistema educativo (pp. 91-110). Santiago de Chile: Facultad de Educación de la Pontificia Universidad Católica de Chile y Oficina Regional para América Latina de UNESCO. 
Gay, G. (2010). Culturally responsive teaching: theory, research, and practice. Nueva York: Teachers College Press.

González, N., Moll, L. C. y Amanti, C. (2005). Funds of knowledge: Theorizing practices in households, communities, and classrooms. Mahwah, NJ: Lawrence Erlbaum Associates.

Gorski, P. C. (2013). Reaching and teaching students in poverty. Nueva York: Teachers College Press.

Howard, G. R. (2006). We can't teach what we don't know: white teachers, multiracial schools (2a ed.). Nueva York: Teachers College Press.

Jones, S. y Vagel, M. D. (2013). Living contradictions and working for change: toward a theory of class-sensitive pedagogy. Educational Researcher, 42(3), 129-141. doi:10.3102/0013189x13481381

Ladson-Billings, G. (1999). Preparing teachers for diversity: Historical perspectives, current trends, and future directions. En L. Darling-Hammond y G. Sykes (Eds.), Teaching as the learning profession: Handbook of policy and practice (pp. 86-123). San Francisco, CA: JosseyBass.

Ladson-Billings, G. (2006). Yes, but how we do it? En J. Landsman y C. W. Lewis (Eds.), White teachers, diverse classrooms. A guide to building inclusive schools, promoting high expectations, and eliminating racism (pp. 29-42). Sterling, VA: Stylus.

López, V., Montecinos, C., Rodríguez, J. I., Calderón, A. y Contreras, J. F. (2012). Enacting solidarity to address peer to peer aggression in schools. En C. Sleeter y E. Soriano (Eds.), Building solidarity across communities of difference in education: International perspectives (pp. 23-44). Nueva York: Teachers' College Press.

Manzi, J., Strasser, K., San Martín, E. y Contreras, D. (2008). Quality of education in Chile. Recuperado de de http://www.ceppe.cl/

Maroy, C. (2004). Regulation and inequalities in European education systems. Final Report. Recuperado de https://www.uclouvain.be/

Merriam, S. B. (2009). Qualitative research: a guide to design and implementation. San Francisco, CA: Jossey-Bass.

Montecinos, C., Ahumada, L., Galdames, S., Campos, F. y Leiva, M. V. (2015). Targets, threats and (dis)trust: the managerial troika for public school principals in Chile. Education Policy Analysis Archives, 23(87), 1-24. doi:10.14507epaav23.2083

Murillo, F. J. y Hernández-Castilla, R. (2011). Hacia un concepto de justicia social. REICE. Revista Iberoamericana sobre Calidad, Eficacia y Cambio en Educación, 9(4), 7-23.

North, C. E. (2008). What is all this talk about "social justice"? Mapping the terrain of education's latest catchphrase. Teachers College Record, 110(6), 1182-1206.

Orfield, G., Kicsera, J. y Siegel-Hawley, G. (2012). E pluribus... separation: deepening double segregation for more students. Recuperado de https://escholarship.org/uc/item/8g58m2v9

Organization for Economic Cooperation and Development. (2013). PISA 2012 results in focus. What 15-year-olds know and what they can do with what they know. París: OECD Publishing.

Organisation for Economic Co-operation and Development. (2015). "Chile”, education policy outlook 2015: Making reforms happen. París: OECD Publishing. doi:10.1787/9789264225442-19-en

Peña-Sandoval, C. (2011). Desigualdad educativa y la necesidad de un enfoque de justicia social en la formación docente. CISMA, Revista del Centro Telúrico de Investigaciones Teóricas, 1, 120. 
Peña-Sandoval, C. (2015). Learning from contexts: A multicase study of secondary preservice teachers in Chile. Tesis doctoral. Universidad de Washington, Estados Unidos. Recuperado de https://digital.lib.washington.edu/researchworks/bitstream/handle/1773/33757/PenaS andoval_washington_0250E_14406.pdf? sequence=1

Riley, K. A. (2013). Leadership of place: Stories from the US, UK and South Africa. Londres: Bloomsbury.

Sleeter C., Montecinos, C. y Jiménez, F. (2016). Preparing teachers for social justice in the context of education policies that deepen class segregation in schools. The case of Chile. En J. Lampert y B. Burnett (Eds.), Teacher education for high poverty schools (pp. 171-191). Cham: Springer.

Sleeter, C. E. (2011). Pedagogías de la equidad en la formación de profesores. En J. Campos, C. Montecinos y A. González (Eds.), Mejoramiento escolar en acción (pp. 15-32). Santiago: Salesianos.

Shields, C. B., Bishop, R. y Mazawi, A. E. (2005). Pathologizing practices: the impact of deficit thinking on education. Nueva York: Peter Lang.

Tedesco, J. C. (2012). Educación y justicia social en América Latina. Buenos Aires: Fondo de Cultura Económica.

Valenzuela, J. P., Bellei, C. y De los Ríos, D. (2014). Socioeconomic school segregation in a market oriented educational system. The case of Chile. Journal of Educational Policy, 29(2), 217-241. doi:10.1080/02680939.2013.806995

Williamson, G. y Montecinos, C. (Eds.). (2011). Educación multicultural. Práctica de la equidad y diversidad para un mundo que demanda esperanza. Talca-Temuco: Universidad de La Frontera.

Zeichner, K. (2009). Teacher education and the struggle for social justice. Nueva York: Routledge.

Zeichner, K. (2010). Rethinking the connections between campus courses and field experiences in college- and university-based teacher education. Journal of Teacher Education, 61, 89-99. doi:10.1177/0022487109347671

Zeichner, K. y Payne, K. (2013). Democratizing knowledge in urban teacher education. En J. Noel (Ed.), Moving teacher education into urban schools and communities: Prioritizing community strengths (pp. 3-19). Nueva York: Routledge.

\section{Breve CV de los autores}

\section{César Peña-Sandoval}

Profesor asociado en la Escuela de Pedagogía de la Pontificia Universidad Católica de Valparaíso donde enseña asignaturas para las carreras de pedagogía en educación primaria y secundaria. Su formación académica es de Profesor de Educación Media en Filosofía y Magister en Curriculum en la Universidad de Chile y Doctor en Curriculum e Instrucción de la University of Washington, Seattle, EEUU. Entre sus publicaciones recientes se encuentran "Venture Philanthropy and Teacher Education Policy in the U.S.: The Role of the New Schools Venture Fund", Teacher College Record, 2015 (en co-autoría) y "Desigualdad educativa y la necesidad de un enfoque de justicia social en la formación docente”, Revista CISMA, 2011. ORCID ID: 0000-0002-5344-0443 Email: cesar.pena@pucv.cl 


\section{Carmen Montecinos}

Psicóloga y Doctora en Psicología Educacional y profesora titular de la Escuela de Psicología de la Pontificia Universidad Católica de Valparaíso donde enseña asignaturas en psicología educacional y educación multicultural. Actualmente es directora ejecutiva del Centro de Liderazgo para la Mejora Escolar. Sus publicaciones más recientes incluyen co-autoría en: "Transitando desde la observación a la acción pedagógica en la práctica inicial: Aprender a enseñar con foco en el aprendizaje del alumnado", aceptado en Estudios Pedagógicos; "Diversidad, modelos de gestión y formación inicial docente: Desafíos formativos desde una perspectiva de justicia social", aceptado en Revista Brasileira de Educação; y "Targets, threats and (dis)trust: The managerial troika for public school principals in Chile", Education Policy Analysis Archives, 2015. ORCID ID: 0000-000 1-8382-1848. Email: carmen.montecinos@pucv.cl 